\title{
FLOW CYTOMETRIC ANALYSIS OF HUMAN ERYTHROCYTES: II. POSSIBLE IDENTIFICATION OF SENESCENT RBC WITH FLUORESCENTLY LABELLED WHEAT GERM AGGLUTININ
}

\author{
Alice Rolfes-Curl, ${ }^{1}$ L. Lazarre Ogden, Geneva M. Omann, ${ }^{2}$ and David AminofF ${ }^{1,3}$ \\ ${ }^{1}$ Institute of Gerontology, ${ }^{2}$ Departments of Surgery and Biological Chemistry, \\ and ${ }^{3}$ Department of Biological Chemistry, The University of Michigan, Ann Arbor, Michigan 48109
}

\begin{abstract}
In the first paper of a series (Gutowski, et al., 1991) we discussed the use of flow cytometry to follow at the cellular level the aging of red blood cells (RBC) in circulation, using fluorescently labelled lectins and goat anti-human-IgG and -IgM. The Coulter Epics 541 was used for those studies. In this report we describe more extensive experiments using the Becton-Dickinson FACScan flow cytometer, and compare the results with those obtained with the Coulter Epics 541. By changing sample conditions from isotonic to hypotonic, compensation for differences of the two instruments was accomplished. We confirmed our previous observations that RBC react very strongly with fluorescein isothiocyanate labelled wheat germ agglutinin (FITC-WGA) and that there is little change in the intensity of fluorescence given by RBC of all sizes with the exception of the smallest. Reactivity with FITC-WGA is markedly decreased in the presence of competitive inhibitors of sialic acid or upon enzymatic removal of sialic acid from RBC. Removal of sialic acid is accompanied by increased reaction with peanut agglutinin (FITCPNA). Flow cytometry was also used to monitor the enrichment of a population of smallest RBC (less than $0.05 \%$ ), isolated from both counterflow centrifugation and the interface obtained from Histopaque separation. These smallest RBC showed low reactivity with FITC-WGA and higher binding of FITC-goat-anti-human-IgG, and -IgM, and therefore represent the most senescent RBC, just prior to their clearance from circulation by the reticuloendothelial system. These observations are in compliance with the hypothesis that physiological desialylation of glycophorin is responsible for clearance of senescent RBC from circulation (Aminoff, 1988).
\end{abstract}

Key Words: aging erythrocytes, autoimmune antibodies, cell size, flow cytometry, human, lectins, senescent erythrocytes, sialidase treatment

\section{INTRODUCTION}

IN THE FIRST PAPER of a series we explored the application of the Coulter Epics 541 flow cytometer to follow changes in red blood cells (RBC) at the cellular level as they age in

Correspondence to: D. Aminoff, Institute of Gerontology, The University of Michigan, 300 North Ingalls Building, Ann Arbor, Michigan 48109-2007.

(Received 29 March 1990; Accepted 20 December 1990) 
vivo (Gutowski et al., 1991). The data obtained supported our hypothesis, that glycophorin or a glycophorin-like molecule on the surface of RBC undergoes time-dependent desialylation in vivo (Aminoff, 1988). Desialylation occurs after appreciable amounts of membrane have been lost as vesicles and accelerates just prior to clearance of RBC from circulation.

While successful in demonstrating the applicability of flow cytometric procedures to the study of RBC, circumstances beyond our control curtailed progress using the Coulter Epics 541, and we were obliged to use the Becton Dickinson FACScan flow cytometer. After an initial adjustment of buffer tonicity we confirmed the observations made with the Coulter Epics, and have better defined the optimal conditions for subsequent flow cytometric analyses of RBC.

Indeed, we used the flow cytometer to monitor counterflow centrifugal separation of RBC (Thompson et al., 1984) and identified the smallest RBC, representing less than $0.05 \%$ of the initial RBC fractionated. These cells epitomized senescent RBC just prior to their physiological clearance from circulation. Senescent RBC are identified by three criteria: (1) smallest RBC, (2) minimal reactivity with fluorescein isothiocyanate (FITC)-wheat germ agglutinin (WGA), and (3) maximal reactivity with autoimmune-IgG and $-\operatorname{IgM}$ as detected with FITC labelled goat-anti-human-IgG and -IgM. Preliminary reports of these studies have already appeared (Ogden et al., 1989; Aminoff et al., 1989).

\section{MATERIALS AND METHODS}

Fluorescein isothiocyanate (FITC) labelled wheat germ agglutinin (WGA), peanut agglutinin (PNA), Histopaque 1077, and phycoerythrin labelled goat-anti-mouse antibody (PEGAM-IgG) were obtained from Sigma (St. Louis, MO). Vibrio cholerae sialidase came from Calbiochem (San Diego, California). $\mathrm{N}, \mathrm{N}^{1} \mathrm{~N}^{11}$-Triacetyl-chitotriose and glycophorin were gifts from Dr. Irwin Goldstein (The University of Michigan, Ann Arbor, MI). Dextran T500 was obtained from Pharmacia (Uppsala, Sweden). Hank's Balanced Salt Solution (HBSS) was obtained as a $10 \mathrm{X}$ concentrate from Gibco (Grand Island, New York). FITC-labelled goat-anti-human-IgG and GAH-IgM came from Kallestad Diagnostic (Austin, Texas). Mouse ascites fluid containing monoclonal antibody to human platelet glycoprotein IIIa (AP-3) was prepared by Newman et al. (1985) and made available by Dr. Curt Hanson. All other reagents were of analytical grade.

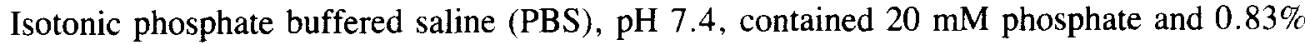
$\mathrm{NaCl}$ (305-310 mOsmol). Hypotonic TRIS buffered saline (hTBS), pH 7.4, contained 3

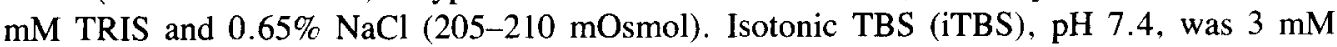
TRIS and $0.87 \% \mathrm{NaCl}(300-305 \mathrm{mOsmol})$. Osmolalities of other buffers were obtained by dilution. Elutriation buffer was as described by Thompson et al. (1984). All buffers used for flow cytometry were filtered through a $0.2 \mu \mathrm{m}$ pore filter.

\section{Human blood}

Human blood from healthy donors was collected by venipuncture into heparinized tubes and stored at $4^{\circ} \mathrm{C}$ for less than 2 hours prior to processing. All donors signed an informed consent for scientific research statement.

\section{Preparation of $R B C$}

Routinely, blood was centrifuged at $250 \times \mathrm{g}$ for $10 \mathrm{~min}$, after which the platelet rich plasma and buffy coat, including some $\mathrm{RBC}$, were removed. The remaining $\mathrm{RBC}$ were 
washed 3 times in 4 volumes of PBS, centrifuged at up to $800 \times \mathrm{g}$ for $10 \mathrm{~min}$, and resuspended in PBS to a hematocrit of 50\%. Washed RBC were stored at $4^{\circ}$ for no more than 2 hours prior to sequential dilution in hTBS (or desired buffer); first to $2.5 \%$ hematocrit and finally to $2.5 \times 10^{6} \mathrm{RBC}$ in $1 \mathrm{ml}$. Cells were then incubated with or without reagents as described below and read directly in the flow cytometer without subsequent washing, unless otherwise stated.

\section{Effect of osmolality}

To determine flow cytometrically the effect of different tonicities on RBC, solutions of the following osmolalities were prepared by dilution of iTBS: 276, 252, 231, 201, and 181 mOsmol.

\section{Reaction of RBC with FITC-WGA}

$0.5 \mu \mathrm{g}\left(1.39 \times 10^{11}\right.$ moles $)$ of FITC-WGA (1.7 moles FITC per mole protein) was added to $2.5 \times 10^{6} \mathrm{RBC}$ in $1 \mathrm{ml}$ hTBS, mixed well and incubated for up to $90 \mathrm{~min}$ at room temperature in the dark.

\section{Separation of blood fractions}

To determine cell size distribution and reactivity with FITC-WGA for all cells found in blood, whole blood was subjected to the following separations: Heparinized blood ( $3.25 \mathrm{ml})$ was diluted 1.4 fold with PBS, layered over $3 \mathrm{ml}$ of Histopaque and centrifuged at $400 \times$ $\mathrm{g}$ for 30 minutes at $20^{\circ} \mathrm{C}$ (Sigma Technical Bulletin No. 1077). Plasma was removed, then the interface, containing mononuclear cells and platelets, was collected. The clear Histopaque layer was discarded leaving RBC and polymorphonuclear cells (PMN) behind. The interface and RBC/PMN fractions were diluted with PBS and washed as described above for RBC. The interface was resuspended in $1 \mathrm{ml}$ PBS and stored at room temperature (RT) while other fractions were prepared, then $154 \mu \mathrm{l}$ was diluted to $1 \mathrm{ml}$ directly in hTBS for flow cytometry. To the RBC/PMN fraction was added 2 volumes of $4.5 \%$ dextran in PBS by a modification of Boyum's procedure (1968). After thorough mixing, the suspension was transferred to a new tube and $\mathrm{RBC}$ allowed to settle at $4^{\circ} \mathrm{C}$. After 1 hour the upper Polymorphonuclear cells (PMN) layer, which also contained $\mathrm{RBC}$, was removed leaving the RBC sediment. Both the PMN and RBC fractions were diluted and washed twice in PBS. For flow cytometry the PMN fraction (hematocrit of $2.5 \%$ ) was diluted directly in hTBS, while the RBC (49\% hematocrit) was serially diluted twice in hTBS as described above. Platelets from $5 \mathrm{ml}$ of whole blood were obtained from platelet-enriched plasma by centrifugation at $800 \times \mathrm{g}$ for $10 \mathrm{~min}$ at RT, washed, and resuspended in $1 \mathrm{ml} \mathrm{PBS}$. For flow cytometry, $100 \mu \mathrm{l}$ of platelet suspension was diluted to $1 \mathrm{ml}$ with hTBS.

\section{Specificity of reaction of $R B C$ with FITC-WGA}

Two approaches were used to establish the specificity of the reaction of FITC-WGA with RBC: (a) inhibition of the reaction with N-triacetylchitotriose or glycphorin, and (b) removal of $R B C$ sialic acid residues with sialidase. 
Treatment of $R B C$ with $N$-triacetyl-chitotriose or glycophorin. To $1 \mathrm{ml}$ of hTBS containing $10^{-5}-10^{-3} \mathrm{M} \mathrm{N}$-acetyl-chitotriose, or $10^{-9}-10^{-6} \mathrm{M}$ glycophorin was added $2.5 \times 10^{6}$ washed RBC and, after $1 \mathrm{~min}, 0.5 \mu \mathrm{g}$ FITC-WGA.

Treatment of RBC with sialidase. Washed RBC $\left(5 \times 10^{9}\right)$ were washed twice in $1 \mathrm{ml}$ HBSS pH 7.1 and resuspended in HBSS to a total volume of $1.5 \mathrm{ml}$ containing from $3.25-$ $157 \mathrm{~m}$ units neuraminidase and incubated for $30 \mathrm{~min}$ at $37^{\circ} \mathrm{C}$. Incubation was terminated by centrifugation, and supernates frozen for later determination of released sialic acid by modified resorcinol method (Jourdian et al., 1971). The RBC were washed three times with PBS, diluted to $50 \%$ hematocrit, and prepared for flow cytometry as above.

\section{Reaction of RBC with FITC-PNA}

$0.5 \mu \mathrm{g}\left(4.17 \times 10^{-12}\right.$ moles $)$ of FITC-PNA (3.0 moles FITC per mole protein) was added to $2.5 \times 10^{6} \mathrm{RBC}$ in $1 \mathrm{ml}$ hTBS, mixed well and incubated for $60 \mathrm{~min}$ at room temperature in the dark.

\section{Counterflow centrifugation of $R B C$}

Several methods were used to enrich for senescent RBC; one of which, counterflow centrifugation, was the most effective. Elutriation was performed according to the method of Thompson et al. (1984), in a Beckman centrifuge No. J21B with an elutriation rotor (JE6) and a Sanderson cell separation chamber (No. 335206), except that equilibration after sample injection was for 5, rather than 10 min prior to collection of the first or "load" (L)fraction, and each fraction was collected for 10 , not 5 , min. Elutriation fractions were centrifuged, and RBC pellets washed in PBS then diluted directly in hTBS at $2.5 \times 10^{5}$ $2.5 \times 10^{6} \mathrm{RBC} / \mathrm{ml}$ for flow cytometry.

Reaction of RBC with FITC-GAH-IgG and -IgM. RBC obtained from counterflow centrifugation were incubated with FITC-GAH-IgG $(8 \mu \mathrm{g}, 2.12 \mathrm{FITC} /$ protein molar ratio) or $-\mathrm{IgM}$ ( $3 \mu \mathrm{g}, 1.87 \mathrm{FITC} /$ protein molar ratio) at $4^{\circ} \mathrm{C}$ for 1 hour prior to analysis.

Reaction of RBC and platelets with AP-3/PE-GAM-IgG and FITC-WGA. The L-fraction obtained from counterflow centrifugation was incubated in $200 \mu \mathrm{l}$ PBS containing $0.4 \mu \mathrm{g}$ platelet specific antibody, AP-3, for $40 \mathrm{~min}$. Cells were centrifuged and washed once with PBS, and resuspended in $100 \mu \mathrm{l}$ of PBS containing $1.14 \mu \mathrm{g}$ PE-GAM-IgG (A568/A280 = 2.3). After $40 \mathrm{~min}$ incubation, cells were centrifuged, washed once in PBS then resuspended, in $1 \mathrm{ml}$ hTBS. For double-labelling experiments FITC-WGA $(0.5 \mu \mathrm{g})$ was added prior to flow cytometric analysis. In spiking experiments $2.5 \times 10^{6}$ platelets prepared from platelet rich plasma were added to L-fraction and incubated as described above.

\section{Flow cytometry and data processing}

Red blood cells were analyzed with a Becton Dickinson FACScan flow cytometer equipped with an argon ion laser emitting at $488 \mathrm{~nm}$ and 15 mwatts. Linear green fluores- 
cence was detected through a $530 \mathrm{~nm}$ filter on FL1 detector and linear red fluorescence detected through a $585 \mathrm{~nm}$ filter on FL2 detector. Voltages were set to assign RBC autofluorescence to channel one. For double-labelling experiments compensation was set to bring fluorescent spillover to background levels. Cell suspensions flowed through the detector at $60 \mu \mathrm{l} / \mathrm{min}$ and approximately 2000 cells per second. Threshold was set on forward lightscatter to exclude dust and small cell debris but not platelets. Lightscatter and fluorescent signals from each of 10000 events, unless otherwise stated, were collected on a Hewlett-Packard series 9000 computer by the Consort 30 program.

Data was acquired in list mode and analyzed by gating five forward lightscatter channels (FSC) at a time. Values obtained for per cent of total events and mean channel of fluorescence (MCF) for each gate were used to identify subpopulations of cells.

\section{RESULTS}

\section{Effect of tonicity (osmolality) on forward lightscatter of $R B C$}

Perhaps the most obvious difference between the two flow cytometers used, Coulter Epics 541 and Becton Dickinson FACScan, is the RBC forward lightscatter (FSC) distribution profile. In the Epics 541, RBC were examined under isotonic conditions (Gutowski et al., 1991). In contrast, the FACScan clearly viewed the flow of ellipsoid, biconcave RBC as essentially two populations of cells under isotonic conditions (276 mOsmol) (Fig. 1A). Reducing buffer tonicity to $201 \mathrm{mOsmol}$ resulted in a monomodal distribution of RBC (Fig. 1C). Similarly, in the presence of FITC-WGA, the FSC histograms showed a bimodal distribution of RBC in isotonic buffer (Fig. 1B), and a monomodal distribution at $201 \mathrm{mOsmol}$ (Fig. 1D). Therefore, in all subsequent experiments hypotonic conditions were used (205$210 \mathrm{mOsmol}$ ).

\section{Reaction of different blood particulate fractions and RBC with FITC-WGA}

Our interest was to determine the extent other particulate blood fractions contribute to the overall profile of RBC distribution. Low background fluorescence was obtained in the absence of FITC-WGA (dot plots of fluorescence vs. FSC) (Figs. 2 A-E) and strong fluorescence obtained in the presence of FITC-WGA (Figs. 2 F-J). As is evident, RBC represent the predominant population of cells in blood and they react very strongly with FITC-WGA (Figs. $2 \mathrm{~F}$ and $\mathrm{G}$ ). Reaction of cells isolated in the interface from Histopaque separation is shown in Figure $2 \mathrm{H}$. Two populations of cells, platelets, and white blood cells were respectively smaller and larger than most RBC and reacted more weakly with FITC-WGA. The absence of these two populations in the whole blood population (Fig. 2 F), indicate the low concentration of these cells relative to RBC.

As previously mentioned (Gutowski et al., 1990), dot plots were found useful as an overview of cell reactions with different probes, but not adequate to demonstrate subtle changes in reactivity of cells of different sizes. This was especially important for the smallest and largest RBC. Figures 3 A-J illustrate the data processed as discussed in Methods for the same samples shown in Figures 2 F-J. Figures $3 \mathrm{~A}-\mathrm{E}$ present reactions in the absence, and Figures $3 \mathrm{~F}-\mathrm{J}$ in the presence of probe. Forward lightscatter of $\mathrm{RBC}$ in absence of any probe, Figures $3 \mathrm{~A}-\mathrm{E}$, indicate that the relative size distribution of blood particles can be identified as platelets at FLS channels 0-25, RBC at channels 25-225, and white blood cells (WBC) overlapping with the largest RBC at channels 150-225. However, neither the platelets nor WBC react as strongly as do RBC with FITC-WGA (Figs. 2, and $3 \mathrm{~F}, \mathrm{G}$, and H). The 

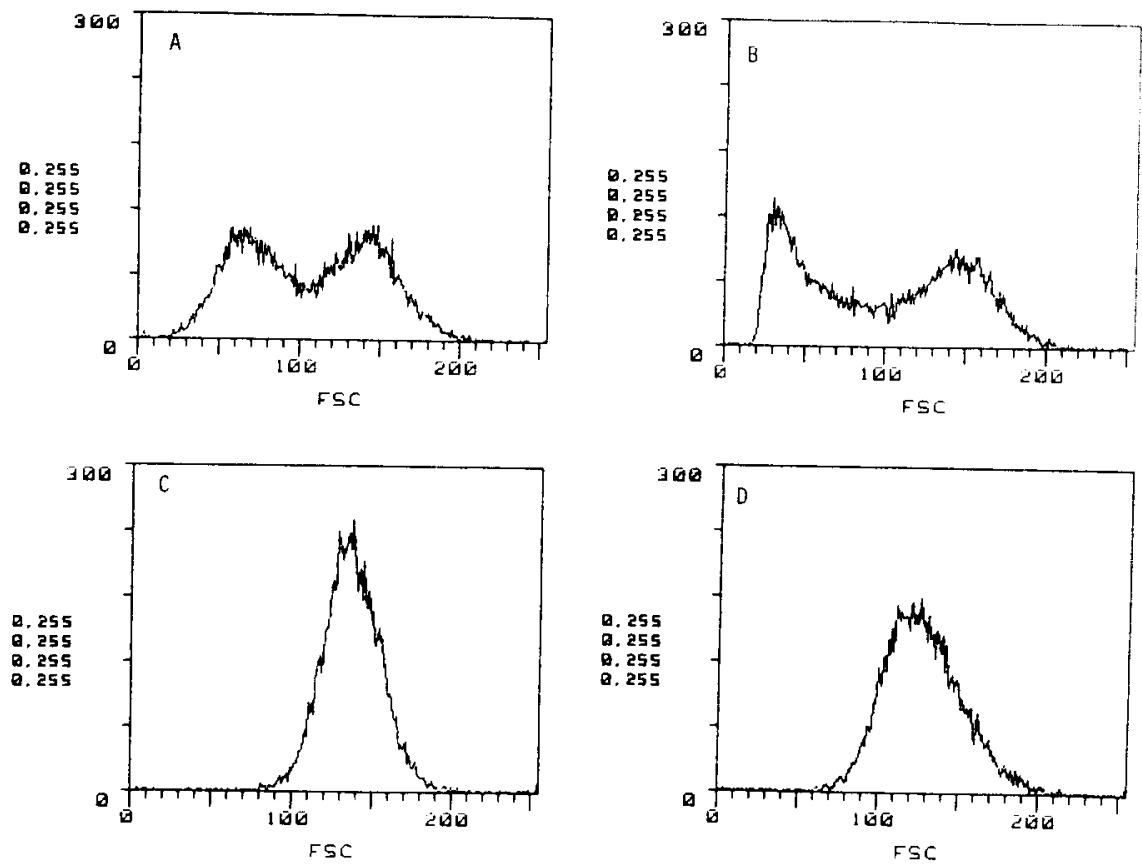

FIG. 1. Effect of tonicity on RBC distribution histograms relating number of events and forward lightscatter (FSC) of $2.5 \times 10^{6} \mathrm{RBC} / \mathrm{ml}$ incubated without $(\mathrm{A}, \mathrm{C}$ ) or with $0.5 \mu \mathrm{g}$ FITC-WGA (B, D); in either iTBS (276 mOsmol) (A, B) or hTBS (201 mOsmol) (C. D).

effect of FITC-WGA on RBC shape is readily seen by comparing cell distribution in Figs 3 $\mathrm{A}, \mathrm{B}$, and $\mathrm{D}$ (in absence), with Figures $3 \mathrm{~F}, \mathrm{G}$, and $\mathrm{I}$ (in presence of lectin). In the presence of plasma, overall intensity of fluorescence with FITC-WGA was less (Fig. 3 F), than with washed RBC (Fig. $3 \mathrm{G}$ ).

As anticipated, the Histopaque interface was rich in platelets and white blood cells (Fig. 3 C). Neither of these populations reacted strongly with FITC-WGA (Fig. $3 \mathrm{H}$ ). A small $(<$ $5 \%$ ) population of cells in the FSC/RBC size range however showed strong reactivity with FITC-WGA (Fig. $3 \mathrm{H}$ ). A sparse population of RBC was confirmed by microscopic examination. This fraction appears to be enriched with senescent RBC.

Cells sedimented through Histopaque consisted predominantly of RBC and PMN cells (Figs. $3 \mathrm{D}$ and I). These two cell types can be partially separated by treatment with dextran (Boyum, 1968) without effectively changing the profiles obtained in the flow cytometer (results not shown). Platelet rich plasma shows a very small contamination with RBC (Fig. 3 E), when observed by forward lightscatter only, but is obvious in the strong signal obtained with FITC-WGA (Fig. $2 \mathrm{~J}$ ). Once again, the smallest $\mathrm{RBC}$ are recognized by their reduced reactivity with FITC-WGA (Fig. $3 \mathrm{~J}$ ).

\section{Reproducibility of data with and without FITC-WGA}

Washed RBC were analyzed in triplicate with and without FITC-WGA. The size distribution and intensity of fluorescence analysis for each of two individuals analyzed in triplicate 

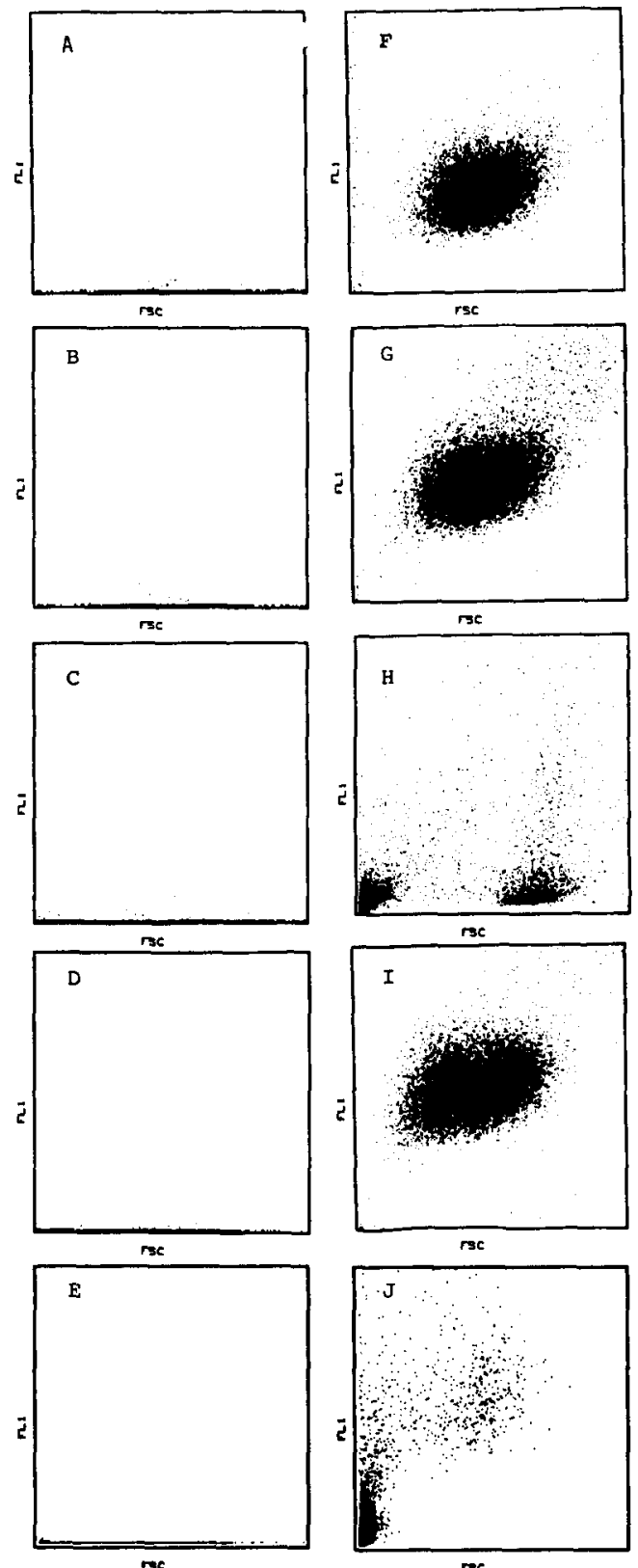

Fig. 2. Reaction of blood particulate fractions with FITC-WGA. Fluorescence (Y-axis) vs. FSC (X-axis) dot plots in the absence (A-E), or presence of $0.5 \mu \mathrm{g}$ of FITC-WGA (F-J), for whole blood (A, F), washed RBC (B, G), Histopaque interface (C, H), Histopaque sediment (D, I), and platelet-RBC (E, J). See Materials and Methods for details.

(mean $\pm \mathrm{SD}$ ) was reproducible (Figs. $4 \mathrm{~A}$ and $\mathrm{B}$ ). A comparison of both individuals in presence of (Fig. $4 \mathrm{C}$ ), and absence of (Fig. $4 \mathrm{D}$ ), FITC-WGA showed greater variability in RBC distribution in the presence of WGA. This variability can be attributed to morpholog- 

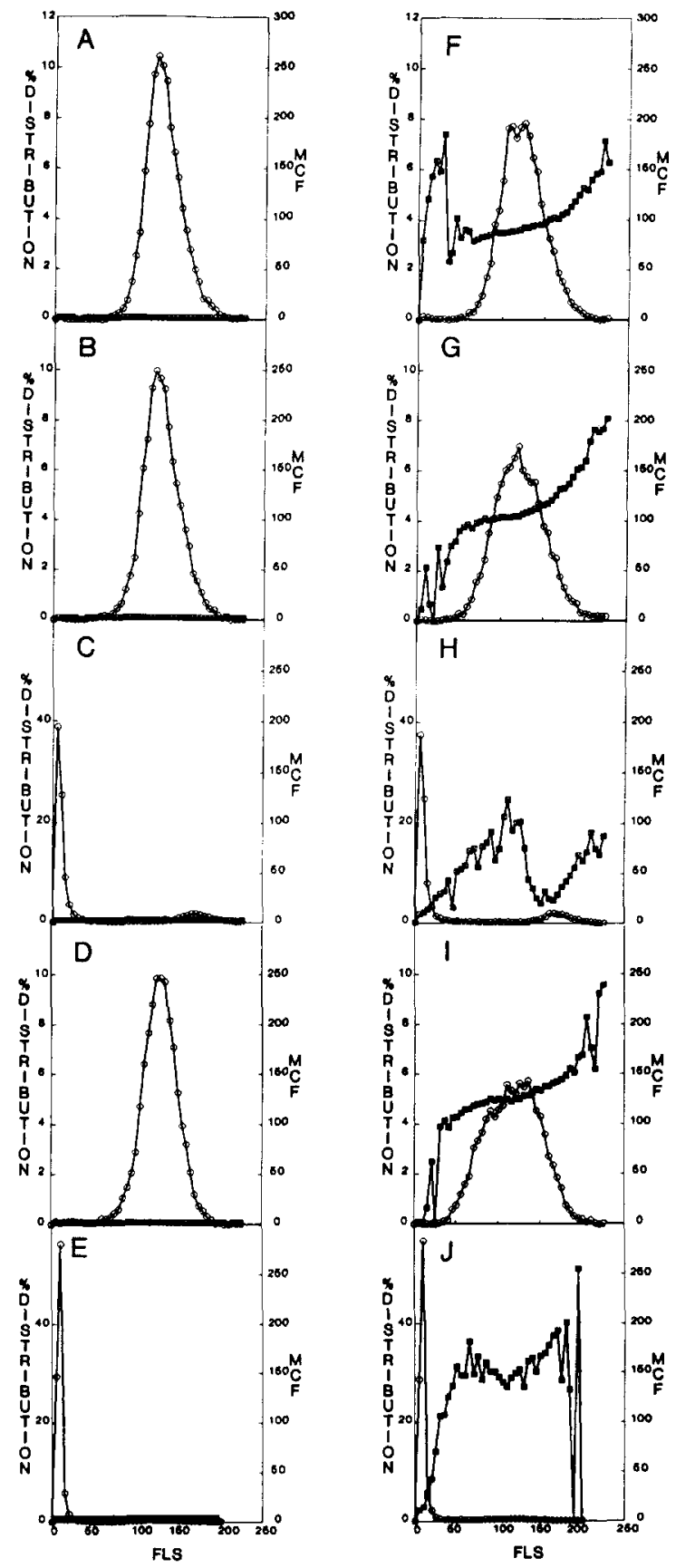

FIG. 3. Reaction of blood particulate fractions with FITC-WGA. Values for $\%$ distribution (O) and mean channel fluorescence (MCF) $(\square)$ were obtained by gating 5 FSC channels at a time, for cells in the absence (A-E) or presence (F-J) of FITC-WGA, from whole blood (A, F), washed $R B C(B, G)$, Histopaque interface $(C, H)$, Histopaque sediment $(D, I)$, and platelet-RBC $(\mathrm{E}, \mathrm{J})$. Note the difference in \% distribution scales for $\mathrm{C}$ and $\mathrm{H}$, and $\mathrm{E}$ and $\mathrm{J}$. 

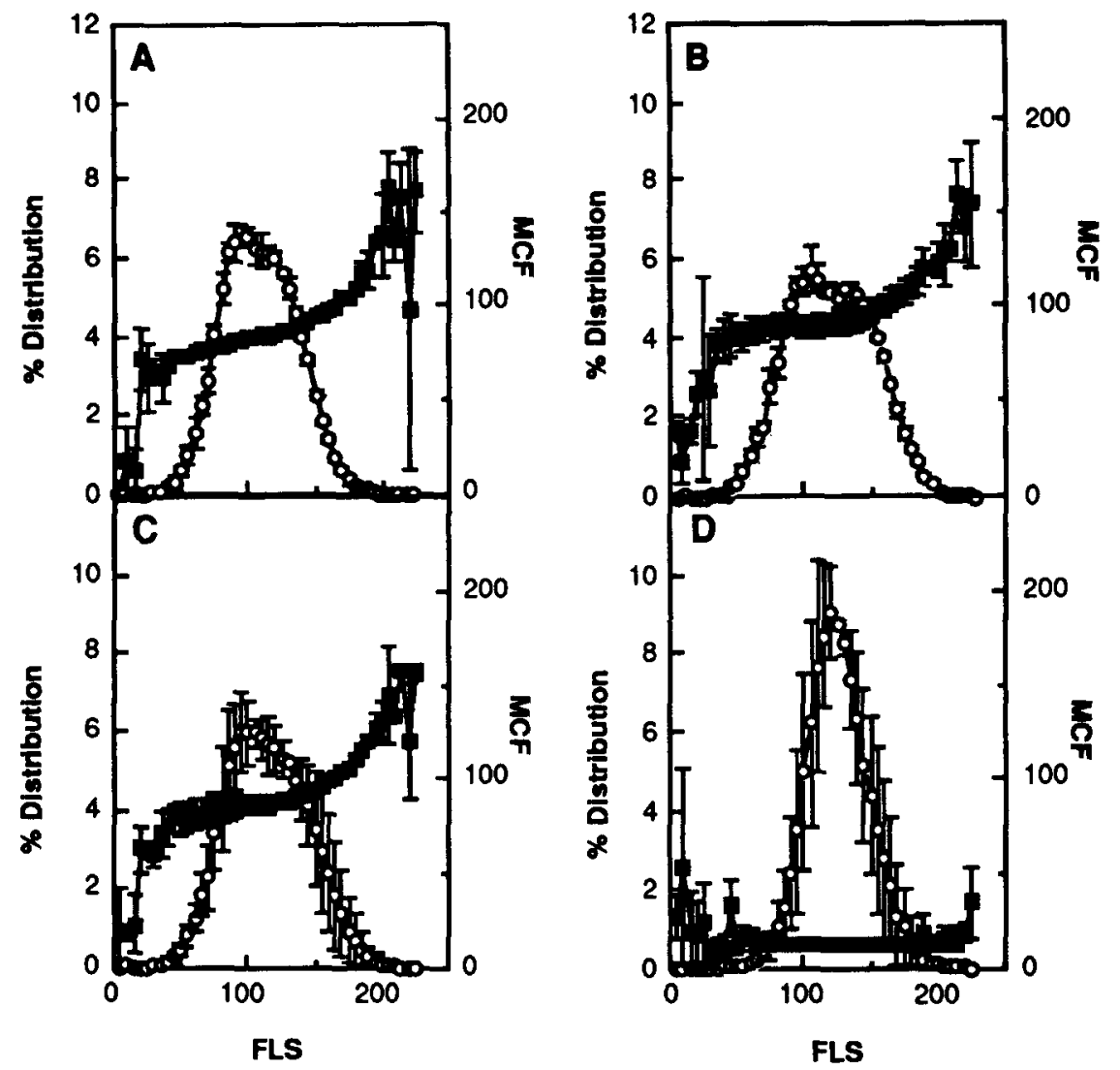

FIG. 4. Reproducibility of results with FITC-WGA. Percent distribution (O) and MCF ( $\square$ ) vs. FLS for $2.5 \times 10^{6}$ washed RBC from two individuals incubated with $0.5 \mu \mathrm{g}$ FITC-WGA (A, B) and for both individuals in the presence (C) and absence of FITC-WGA (D). Mean values $\pm S D$ are plotted for $n=3$ determinations.

ical changes caused by WGA, the dynamics of which will be discussed in a subsequent publication.

\section{Titration of FITC-WGA binding to RBC}

Two distinct experiments were performed: (a) number of RBC was kept constant (at 2.5 $\times 10^{6}$ ), and amount of FITC-WGA varied from 0-1 $\mu \mathrm{g}$ (Table 1), and (b) amount of FITCWGA was kept constant (at $0.5 \mu \mathrm{g} / \mathrm{ml}$ ), but number of RBC varied from $2.5 \times 10^{4}$ to 2.5 $\times 10^{7}$ (Table 2).

As is apparent from Table 1, mean channel of fluorescence (MCF) is proportional to the concentration of FITC-WGA. The concentration of $0.5 \mu \mathrm{g} / \mathrm{ml}$, routinely used, was sufficiently sensitive to detect different reactivities of cells with FITC-WGA. When the amount of FITC-WGA was kept constant, MCF changed with number of RBC probed, and attained maximal MCF with $2.5 \times 10^{5} \mathrm{RBC}$ (Table 2). Incubation with FITC-PNA (Table 4, without sialidase treatment) served as a nonbinding fluoresceinated protein control having the same MCF as obtained in the absence of any FITC probe. 
Table 1. Titration of Fitc-wga binding to $2.5 \times 10^{\circ} \mathrm{RBC}$

\begin{tabular}{lcr}
\hline $\begin{array}{l}\text { FITC-WGA } \\
(\mu g)\end{array}$ & $M C F$ & $C V$ \\
\hline 1.0 & 125.67 & 7.1 \\
0.5 & 81.31 & 18.3 \\
0.25 & 46.58 & 29.4 \\
0.125 & 26.79 & 18.8 \\
0.0625 & 13.58 & 18.3 \\
0. & 4.0 & 4.4 \\
\hline
\end{tabular}

Washed RBC were incubated with different amounts of FITC-WGA. MCF and CV (coefficient of variance) values are for all 10000 events counted.

Inhibition of reaction of $R B C$ with FITC-WGA by $N$-triacetyl-chitotriose, and glycophorin

The effect of N-triacetyl-chitotriose, a strong competitive inhibitor of sialic acid (Allen et al., 1973), on binding of FITC-WGA to RBC analyzed either by sequential gating of five forward lightscatter channels (FSC) or by MCF values for all 256 FSC channels is presented in Figure 5 and Table 3, respectively. N-triacetyl-chitotriose at $10^{-3} \mathrm{M}$ completely inhibited attachment of FITC-WGA to RBC. Inhibition with $10^{-5} \mathrm{M}$-triacetyl-chitotriose resulted in a curve parallel to that obtained in the absence of inhibitor. As would be expected, glycophorin at a concentration of $10^{-6} \mathrm{M}$ almost completely inhibited the reaction of FITCWGA with RBC (Table 3).

Effect of sialidase treatment of RBC on their reactivity with FITC-WGA and -PNA

In two experiments, decrease in fluorescence after sialidase treatment gave a series of parallel curves, with no preferential loss of sialic acid from the smallest as compared to the largest cells (Figure 6) (data from one experiment). Table 4 summarizes both MCF decrease with FITC-WGA and increase with FITC-PNA as a function of decreasing sialic acid content.

Table 2. Titration of RBC With $0.5 \mu \mathrm{G}$ Fitc-wga

\begin{tabular}{lrr}
\hline $\begin{array}{l}\text { Number } \\
\text { of } R B C\end{array}$ & $M C F$ & $C V$ \\
\hline $2.5 \times 10^{4}$ & 109.17 & 20.9 \\
$2.5 \times 10^{5}$ & 120.29 & 16.2 \\
$2.5 \times 10^{6}$ & 81.31 & 18.3 \\
$2.5 \times 10^{7}$ & 17.46 & 30.9 \\
\hline
\end{tabular}

Different concentrations of washed RBC were incubated with FITCWGA. MCF and CV values are for all 10000 events counted. 


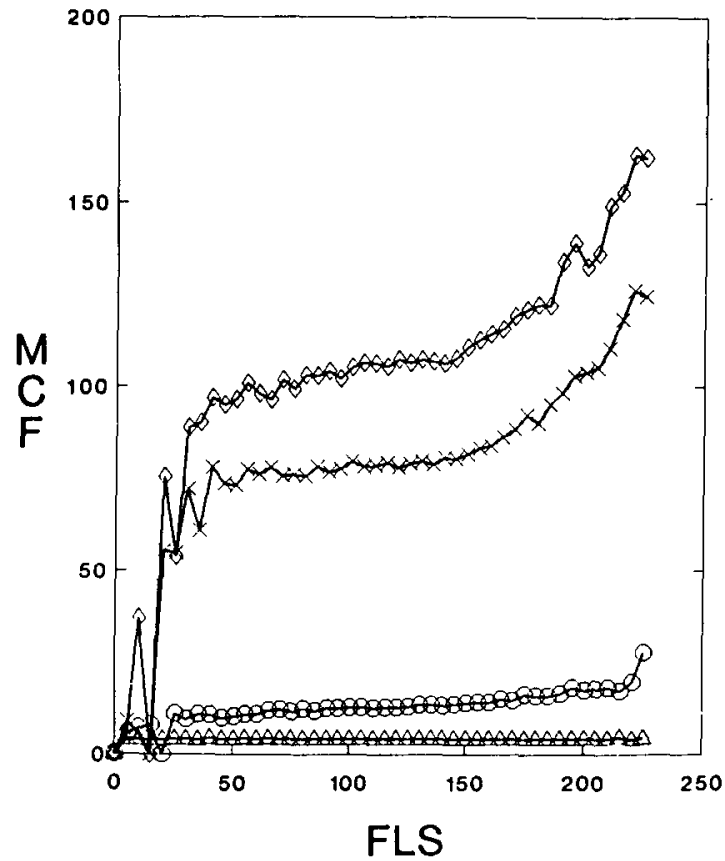

FIG. 5. Inhibition of FITC-WGA binding with $\mathrm{N}$-acetyl-chitotriose. $2.5 \times 10^{6} \mathrm{RBC}$ were incubated without $(\diamond)$ or with: $10^{-5} \mathrm{M}(\times), 10^{-4} \mathrm{M}(O), 10^{-3} \mathrm{M}(\triangle) \mathrm{N}$-acetyl-chitotriose prior to addition of $0.5 \mu \mathrm{g}$ FITC-WGA.

\section{Counterflow centrifugation and characterization of senescent $R B C$}

Using a modified procedure of that described by Thompson (1984) nine fractions were isolated and examined in the flow cytometer without and with FITC-WGA (Figures 7 A-I). The first fraction, the load or L-fraction (Fig. $7 \mathrm{~A}$ ), contained platelets and $0.05 \%$ of the

TABLE 3. INHIBITION OF FITC-WGA BINDING TO RBC

\begin{tabular}{lccccc}
\hline & $\begin{array}{c}\text { Inhibitor } \\
\text { concentration } \\
\text { Inhibitor }\end{array}$ & \multicolumn{2}{c}{$-($ FITC-WGA $)$} & \multicolumn{2}{c}{$+($ FITC-WGA $)$} \\
\hline N-Acetyl- & 0 & $M C F$ & $C V$ & $M C F$ & $C V$ \\
Chitotriose & $10^{-5}$ & 4.02 & 15.2 & 108.5 & 22.6 \\
& $10^{-4}$ & 4.01 & 4.9 & 81.19 & 26.2 \\
Glycophorin & $10^{-3}$ & 4.01 & 5.1 & 13.40 & 40.2 \\
& 0 & & & 4.2 & 10.7 \\
& $10^{-9}$ & 4.01 & 7.6 & 87.59 & 19.9 \\
& $10^{-8}$ & 4.01 & 7.8 & 93.70 & 19.9 \\
& $10^{-7}$ & 4.00 & 2.1 & 83.23 & 20.9 \\
& $10^{-6}$ & 4.01 & 6.8 & 51.31 & 29.0 \\
& & 4.00 & 3.9 & 10.93 & 72.2 \\
\hline
\end{tabular}

$2.5 \times 10^{6}$ washed RBC were incubated with inhibitor for one minute prior to addition of $0.5 \mu \mathrm{g}$ FITC-WGA. 


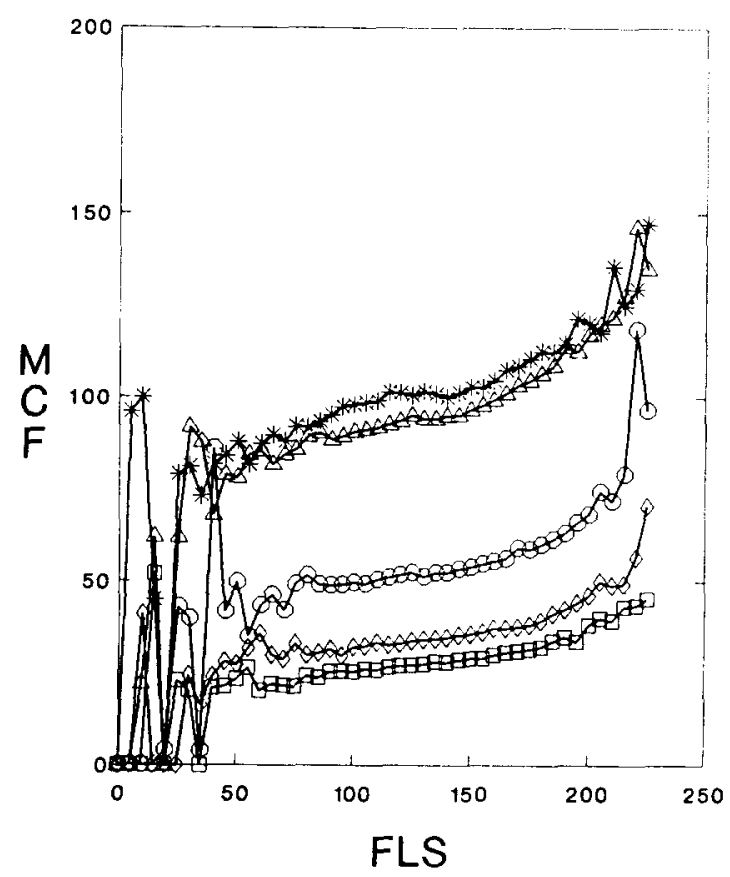

FIG. 6. Reactions of enzymatically desialylated RBC with FITC-WGA. $2.5 \times 10^{6} \mathrm{RBC}$ were incubated with $0.5 \mu \mathrm{g}$ FITC-WGA after treatment with $157(\square), 26(\diamond), 13(\bigcirc), 3.25(\triangle)$, or $O$ (*) m units of sialidase.

RBC fractionated. This value was determined by visual comparison to two-fold serial dilutions of known concentration of unseparated $\mathrm{RBC}$, and by count rate obtained from the flow cytometer. As can be seen from cell size distribution, RBC in Figure 7 A are of smallest size. Subsequent fractions (Figure 7 B-I), showed both an increase in mean cell size and broader size distribution. Thus, with the exception of the load fraction (Figure $7 \mathrm{~A}$ ), counterflow centrifugation did not give clear separation of populations of RBC of different size. In presence of FITC-WGA, load fraction RBC viewed in SSC vs. FSC dot plot (Fig. $8 \mathrm{~A}$ ) showed two cell populations, platelets, and smallest RBC; while FLI vs. FSC dot plot (Fig. $8 \mathrm{~B}$ ), showed decreasing fluorescence of RBC with decreasing cell size. Platelets reacted

Table 4. Sialidase treatment of RbC

\begin{tabular}{|c|c|c|c|c|c|c|c|}
\hline \multirow{2}{*}{$\begin{array}{l}\text { Sialidase } \\
\text { ( } m \text { units) }\end{array}$} & \multirow{2}{*}{$\begin{array}{c}\text { \% Remaining } \\
\text { Sialic Acid }\end{array}$} & \multicolumn{2}{|c|}{ Control } & \multicolumn{2}{|c|}{ FITC-WGA } & \multicolumn{2}{|c|}{$F I T C-P N A$} \\
\hline & & $M C F$ & $C V$ & $M C F$ & $C V$ & $M C F$ & $C V$ \\
\hline 157.0 & 0. & 3.99 & 5.9 & 28.67 & 19.5 & 56.54 & 25.2 \\
\hline 26.0 & 18. & 4.00 & 9.6 & 35.30 & 20.8 & 18.26 & 19.0 \\
\hline 13.0 & 37. & 3.99 & 8.0 & 53.81 & 22.8 & 9.02 & 22.9 \\
\hline 3.3 & 81. & 3.99 & 6.5 & 96.10 & 18.0 & 3.91 & 19.1 \\
\hline 0.0 & 100. & 3.99 & 5.1 & 101.41 & 17.8 & 3.90 & 29.1 \\
\hline
\end{tabular}

$2.5 \times 10^{6}$ sialidase treated $\mathrm{RBC}$ were incubated with $0.5 \mu \mathrm{g}$ FITC-WGA or FITC-PNA. 

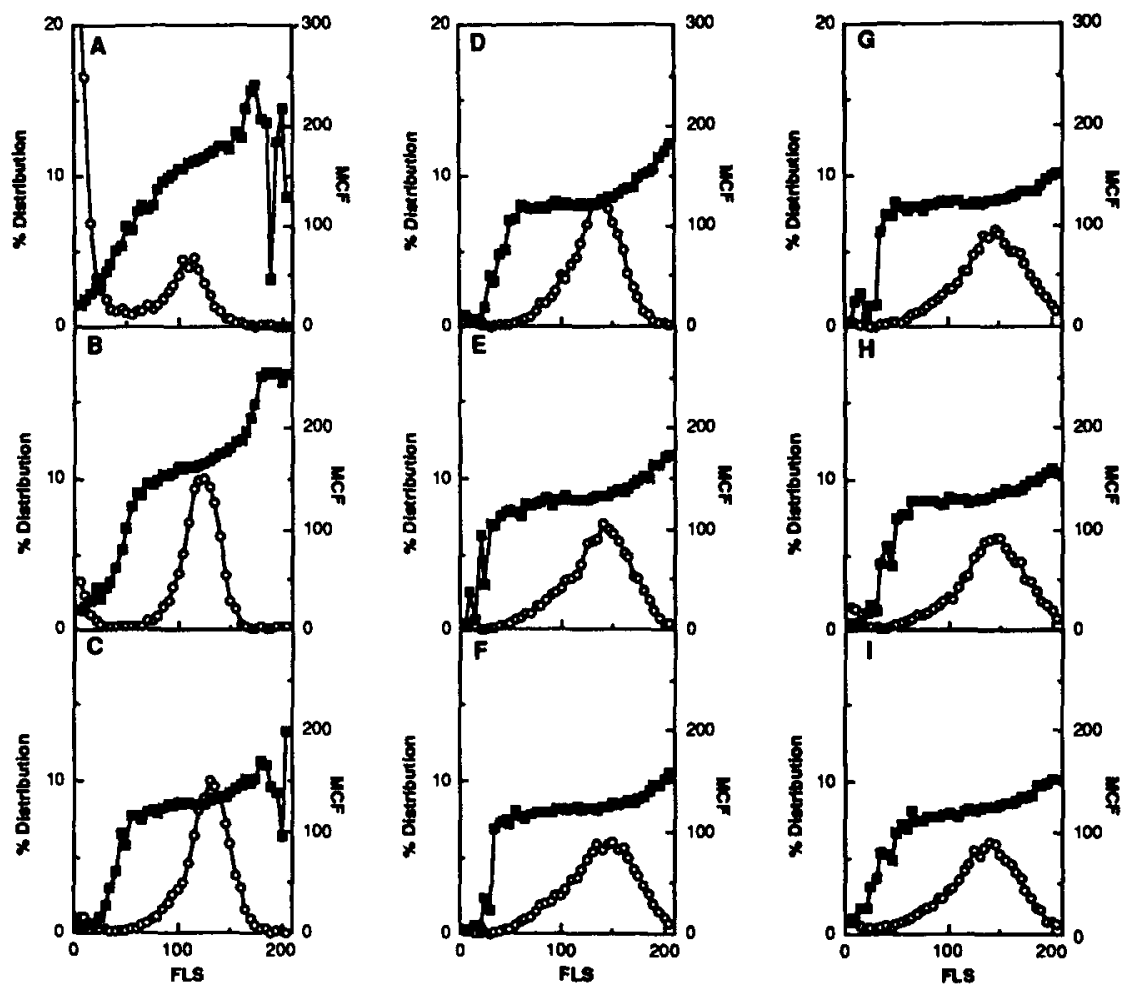

FIG. 7. Reaction of RBC with FITC-WGA after RBC fractionation by counter flow centrifugation. $1 \times 10^{9} \mathrm{RBC}$ were separated into $10 \mathrm{~min}$ fractions collected at flow rates of $4(\mathrm{~A}), 7(\mathrm{~B})$, $8(\mathrm{C}), 9(\mathrm{D}), 10(\mathrm{E}), 11(\mathrm{~F}), 12(\mathrm{G}), 13(\mathrm{H}) \mathrm{ml} / \mathrm{min}$, respectively. $\mathrm{RBC}$ remaining in elutriation chamber are shown in (I). All fractions were washed, resuspended in hTBS prior to incubation with $0.5 \mu \mathrm{g}$ FITC-WGA. Percent distribution (O) and MCF ( $\square$ ).

weakly with FITC-WGA. Further analysis of the load fraction showed reaction of smallest RBC, but not platelets, with FITC-GAH-IgG and -IgM (Figs. 9 B, C). Reactivity with FITC-GAH-IgG and -IgM implies that these smallest RBC are partially covered with autoimmune $-\operatorname{IgG}$ and $-\operatorname{IgM}$.

\section{Characterization of particles in L-Fraction}

In subsequent experiments we removed as many platelets as possible prior to fractionation by counterflow centrifugation. In single-labeling experiments with mouse monoclonal antibody to human platelet glycoprotein IIIa (AP-3) few platelets were found in the L-fraction. In double-labelling experiments strong fluorescence with FITC-WGA and weak fluorescence with AP-3/PE-GAM-IgG were obtained (Figs. $10 \mathrm{~A}$ and C). The FITC-WGA pattern was unchanged when AP-3/PE-GAM-IgG-reacting particles were gated out (Fig. $10 \mathrm{~B}$ ). To verify that platelets could be detected in the presence of $\mathrm{RBC}$, double-labelling was performed using a platelet-spiked L-fraction. Strong FITC-WGA and AP-3/PE-GAM-IgG signals were obtained (Figs. $10 \mathrm{E}$ and G). Again, when AP-3/PE-GAM-IgG-reacting particles were gated 

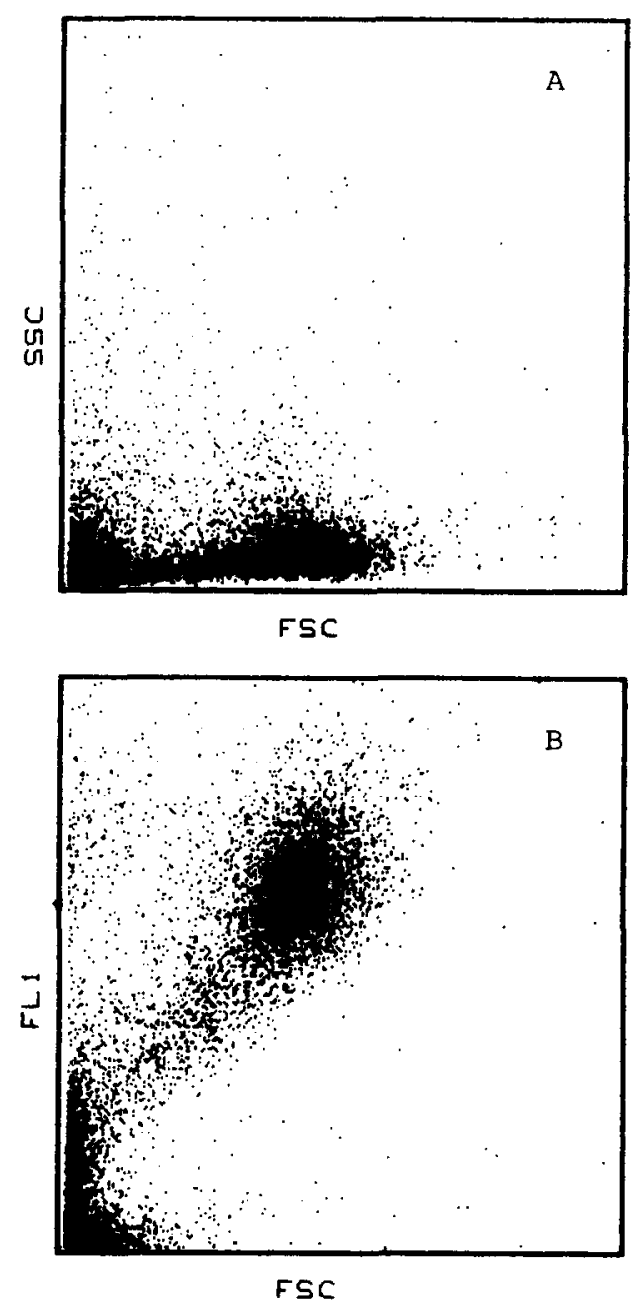

FIG. 8. Dot plots of the smallest RBC obtained by counterflow centrifugation. Side scatter vs. forward lightscatter (A), and fluorescence vs. forward lightscatter in presence of $0.5 \mu \mathrm{g}$ FITCWGA (B). Data for channels 0-255 for all parameters.

out the FITC-WGA profile was unchanged (Fig. $10 \mathrm{~F}$ ). Effectiveness of gating out the PElabelled particles is shown in Figures $10 \mathrm{D}$ and $\mathrm{H}$.

\section{DISCUSSION}

Most investigators have been reticent to apply flow cytometric procedures to the study of $\mathrm{RBC}$ because of the known age-related changes in erythrocyte shape. In our previous report (Gutowski et al., 1991) we, like Dockter et al. (1986) and Jennings et al. (1985), found that RBC could be analyzed in isotonic condition in the Coulter Epics. Furthermore, we demonstrated that smallest and largest cells did show the properties anticipated for oldest and youngest RBC in their reactivity with FITC-WGA and FITC-labelled goat-anti-humanIgG, and -IgM. 


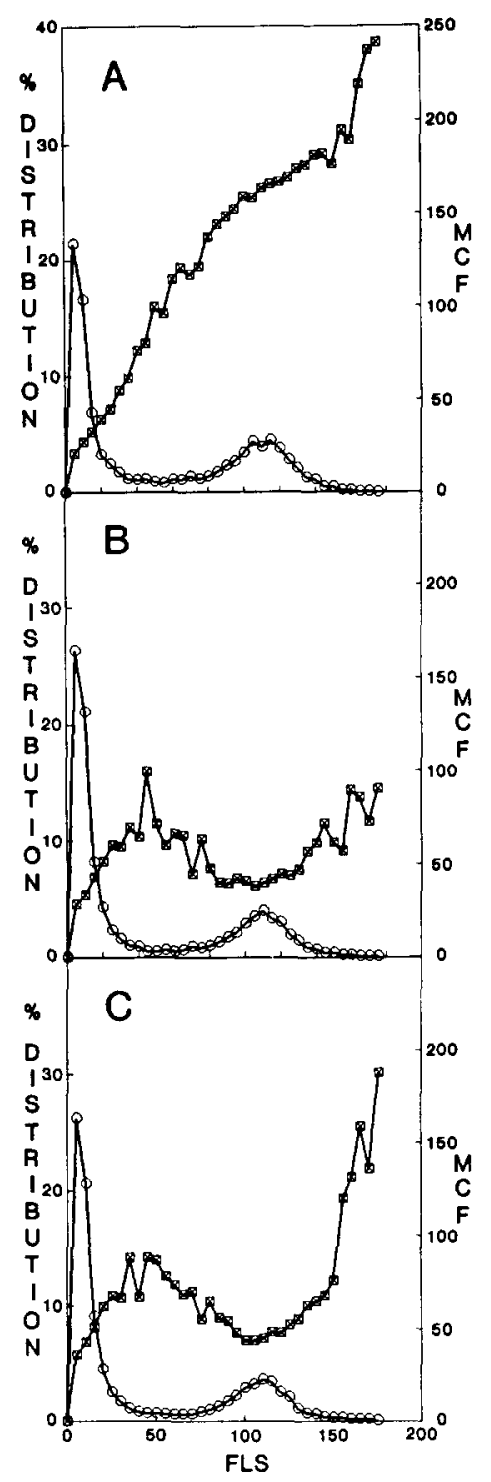

FIG. 9. Reaction of the smallest RBC with FITC-GAH-IgG and FITC-GAH-IgM. Load fraction RBC obtained by counterflow centrifugation were incubated with $0.5 \mu \mathrm{g}$ FITC-WGA (A), $8 \mu \mathrm{g}$ FITC-GAH-IgG (B), or $3 \mu \mathrm{g}$ FITC-GAH-IgM (C). Percent distribution (O) and MCF (D).

In this study using the BD FACScan we immediately learned that lightscatter analysis of $\mathrm{RBC}$ in isotonic buffer gave a disperse bimodal distribution in contrast to a guassian distribution obtained with the Coulter Epics. This difference is attributed to orientation of ellipsoid biconcave RBC in flow cells of different geometry and forward scatter detectors. The FACScan uses a solid state detector and measures signal height, whereas the Epics uses a photomultiplier tube and integrates signals. Although previous investigators had increased sphericity of RBC isovolumetrically using SDS/albumin and a trace of glutaraldehyde (Kim 

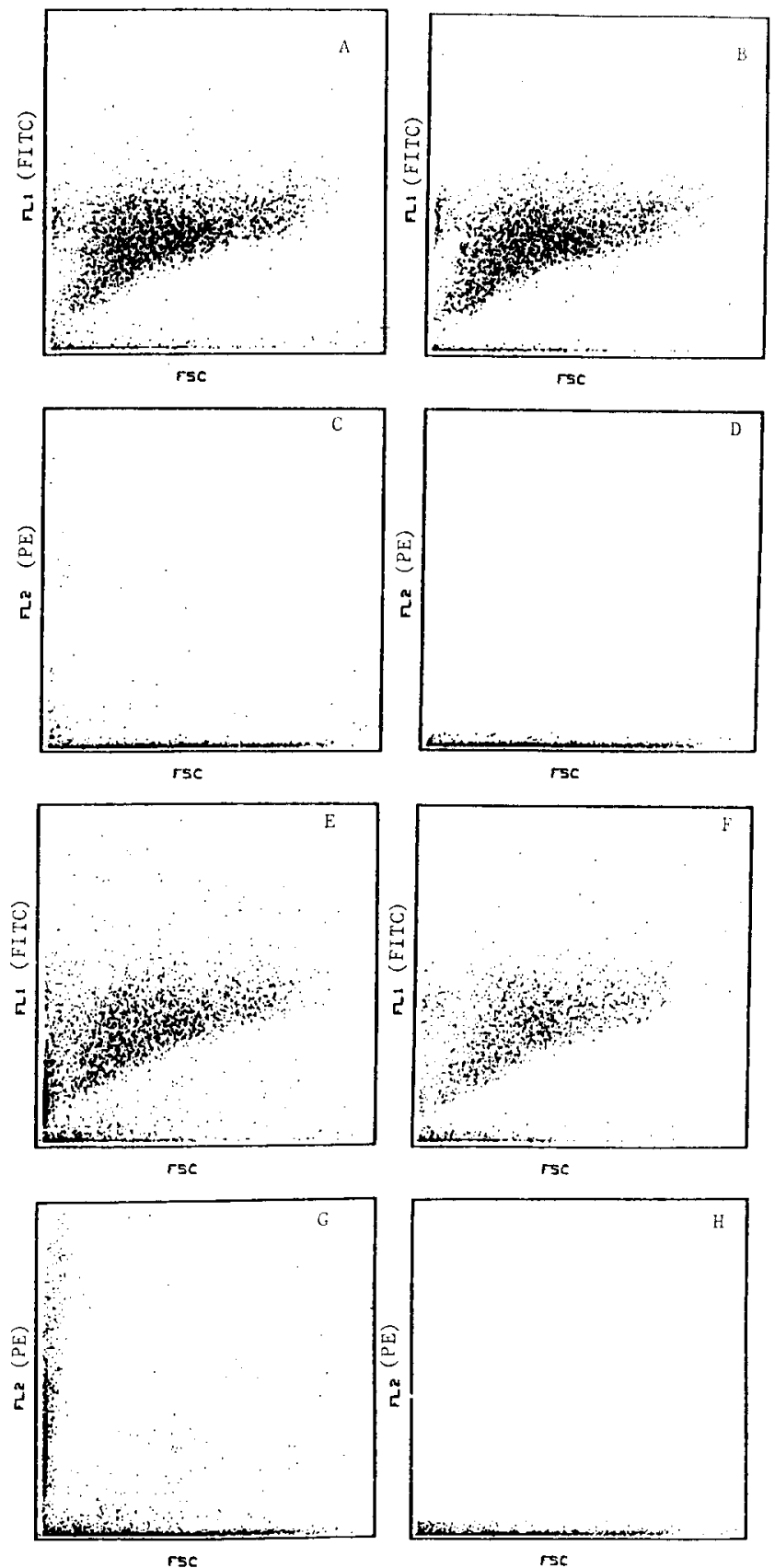

FIG. 10. Characterization of particles in L-fraction. Dot plots of L-fraction, containing $5 \times 10^{5}$ RBC (A-D), and L-fraction spiked with $2.5 \times 10^{6}$ platelets $(\mathrm{E}-\mathrm{H})$ labelled with AP-3/PE-GAMIgG and FITC-WGA. A. FITC signal for L-fraction; B. FITC signal (for same samples as A) live gated on FITC to exclude PE-labelled particles; C. PE signal (for same sample as A); D. PE signal (for same sample as A) live gated on FITC to exclude PE; E. FITC signal for platelet spiked L-fraction; F. FITC signal (for same sample as E) live gated on FITC; G. PE signal (for same sample as E); H. PE signal (for same sample as E) live gated on FITC. 5000 events were collected for A, B, C, D, E, and G; 2500 events for F and H. 
et al., 1983), or by fixing with dimethylsuberimide (Langois et al., 1985) we wished to continue our studies in the absence of detergents or fixatives. Decreasing tonicity of buffers in which RBC were suspended decreased forward lightscatter bimodality, and at 200-210 mOsmol cells appeared as a homogeneous monomodal peak with a "gaussian" distribution. This compensation, however, was not without pitfalls. Using blood from one individual in pilot studies we noted minimal effects of FITC-WGA on cell shape. In reproducibility studies with RBC from different individuals, each sample analyzed in triplicate, we noted changes in cell shape in the presence of FITC-WGA. Change in shape was time-dependent (over $90 \mathrm{~min}$ ) while maximum fluorescence was attained in 5 minutes. The effect of WGA on RBC shape had been reported previously (Anderson and Lovrien, 1980) but probably would not have been detected by flow cytometry if fixatives were used.

Dockter et al. (1986) and Jennings et al. (1985) previously established MCF/FLS (mean channel of fluorescence vs. forward lightscatter plot) as a useful means of following changes in $\mathrm{RBC}$ surface markers with change in cell size. We confirmed its usefulness in our previous study using FITC-labelled WGA, GAH-IgG, and GAH-IgM and the Coulter Epics 541 flow cytometer (Gutowski et al., 1991). In this study, using the BD FACScan, we again confirm the utility of this analytical method despite greater instrument sensitivity to changes in cell shape. Indeed, it provides us with another way to study signal transduction of membrane ligand reactions.

Wheat germ agglutinin has dual specificity: it recognizes both sialic acid and $\mathrm{N}$-acetylglucosamine residues. Specificity of FITC-WGA for sialic acid residues on RBC surface was again substantiated by inhibition and enzymatic desialylation studies. The binding of FITCWGA to RBC was completely inhibited by $10^{-3} \mathrm{M}$ N-triacetyl-chitotriose, a trisaccharide of $\mathrm{N}$-acetylglucosamine residues, and by $10^{-6} \mathrm{M}$ glycophorin A that contains 31 sialic acid residues per molecule. This is a significant difference in inhibiting potency and reflects the primary reactivity of FITC-WGA with sialic acid residues. In addition, Figures 2 F, G, and $3 \mathrm{~F}$ and $\mathrm{G}$ indicate that plasma contains soluble sialoglycoconjugates that compete with $\mathrm{RBC}$ for FITC-WGA. Enzymatic removal of sialic acid did not inhibit all binding of FITC-WGA, whereas inhibition with triacetyl-chitotriose did. No doubt removal of sialic acid residues results in exposure of $\mathrm{RBC}$ erythroglycans, $\mathrm{N}$-acetylglucosamine containing glycoproteins which can react with FITC-WGA (Bhavanadan et al., 1979; Ivatt et al., 1986). Partial removal of sialic acid residues, however, did not reveal a difference in the rate of desialylation (Fig. 6) between small and large RBC as we previously observed with the Coulter Epics 541 (Gutowski et al., 1991).

Although complete desialylation of RBC with Vibrio sialidase did not completely inhibit binding of FITC-WGA, the resulting aRBC reacted maximally with FITC-PNA, indicating exposure of galactose $\beta(1,3)-\mathrm{N}$-acetylgalactosaminyl disaccharide residues. Binding of FITC-PNA to asialo-RBC was also accompanied by a change in RBC shape. Interestingly, removal of only $20 \%$ of the total $\mathrm{RBC}$ sialic acid susceptible to sialidase did not result in perceptible fluorescence with FITC-PNA. Moreover, there is a nonlinear proportionality between the loss of sialic acid and consequent reactivity with PNA (Table 4). The data suggest that the presence of PNA binding sites on RBC surface does not automatically imply accessibility to PNA.

While it was difficult to conclude that there is a significant population of small RBC in unseparated RBC that have a diminished reactivity with FITC-WGA (Figs. $2 \mathrm{G}$ and $4 \mathrm{~A}, \mathrm{~B}$, and $\mathrm{C}$ ), enrichment of smallest RBC population left no doubt, as best seen in Figure $7 \mathrm{~A}$. 
RBC separated according to size by counterflow centrifugation either by the method of Thompson (1984) or Vaysse (1988) gave RBC in the load fraction with properties associated with oldest cells (Figs. $7 \mathrm{~A}, 8 \mathrm{~A}$ and $\mathrm{B}$ ). The L-fraction RBC represented $0.05 \%$ of the $\mathrm{RBC}$ fractionated and were contaminated with some platelets. Presumably they were rejected with platelets by both Thompson and Vaysse.

The smallest RBC (FLS channels 25-75), representing less than $0.01 \%$ of RBC fractionated, react minimally with WGA but more strongly with GAH-IgG and -IgM (Fig. 9). We believe represent the oldest $\mathrm{RBC}$ in circulation just prior to their clearance. They are indeed the smallest RBC and not membranes from lysed RBC for three reasons: (a) Figs. $8 \mathrm{~B}$ and 9 A show a smooth continuum of decreasing reactivity with FITC-WGA, while some of those cells show an increased reactivity with FITC-GAH-IgG and -IgM (Figs. 9 B and 9 C), (b) membranes of lysed L-fraction RBC are found at FLS 0-25 and react with FITC-WGA, and (c) are not a result of exposure to hypotonic buffer because they are not evident in all elutriation fractions or in unseparated RBC. Furthermore, recent experiments with monoclonal antibodies to human glycophorin have shown that these particles are erythroid in origin.

However, the fluorescence of particles seen in channel FSC 25-75 of L-fraction (Fig. 8 $B$ ) could also be interpreted as an increasing reactivity of platelets and platelet aggregates with FITC-WGA rather than decreasing reactivity with decreasing $\mathrm{RBC}$ size. Experiments with AP-3 showed that the decrease of FITC-WGA fluorescence in the L-fraction is not due to platelets or platelet aggregates but to small RBC (Fig. 10), presumably senescent-RBC.

It is interesting to note that a similar population of small $\mathrm{RBC}$ was detected in other platelet-containing fractions, that is (Fig. $3 \mathrm{H}$ ), which react with FITC-WGA, and reactivity falls with decrease in cell size. Enrichment of senescent-RBC in platelet containing fractions is a startling finding, since it appears to be inconsistent with previous observations attributing the greatest density to the oldest RBC. A recent observation of Heldrup (1990) reports on the contamination of Ficoll-Paque-separated leucocytes with erythroid cells as detected with an antibody to glycophorin $\mathrm{A}$.

In the previous manuscript (Gutowski et al., 1991) we established that there was a difference between the smallest and the rest of the RBC population. This difference - decreased reactivity with WGA, and increased reactivity with goat-anti-human-IgG and $-\operatorname{IgM}$ - is compatible with our hypothesis that physiological desialylation of glycophorin is responsible for clearance of senescent $\mathrm{RBC}$ from circulation and therefore identifies the smallest as the most senescent RBC just prior to their clearance. In this report (concentrating on the reaction of RBC with WGA) we confirmed on B-D FACScan our observations previously made with Coulter Epic (Gutowski et al., 1991). More detailed study of RBC reactions with WGA confirmed that changes in sialic acid content on the surface of RBC were readily detected. Furthermore, decreased reactivity of smallest RBC with WGA is gradual and more definitive in populations enriched for small $R B C$.

Recently there has been a surge of interest in RBC aging and the mechanism by which senescent-RBC are cleared from circulation. There are several contending hypotheses (Aminoff, 1989). All agree that RBCs change in size, shape, and rigidity of cell membrane as they age in vivo. Of even greater importance is agreement that there is a molecular basis for aging and sequestration. There is however considerable disagreement with regard to the putative molecule invoked as signal. Is it a degradation product of Band 3 (Kay, 1978), or a glycolipid terminating in an $\alpha$-galactosyl residue (Galili et al., 1984), or as is our belief, that it involves desialylation of glycophorin? With approaches presented here it is now pos- 
sible to address, in a new way, the different hypotheses proposed for the clearance of effete erythrocytes.

Acknowledgment - We gratefully appreciate the helpful discussion we have had with Drs. Jerry L. Hudson, David Fox, Philip McCoy, and Robert Todd, data processing and graphic skills of Ms. Heather K. Clark, and secretarial assistance of Ms. Fran A. Gruda. This research was supported by the National Institutes of Health grant \#AG08018

\section{REFERENCES}

ALLEN, A.K., NEUBERGER, A,, and SHARON, N. The purification, composition, and specificity of wheat germ agglutinin. Bioch. J. 131, 155-162, 1973.

AMINOFF, D. The role of sialoglycoconjugates in the aging and sequestration of red cells from circulation. Blood Cells 14, 229-257, 1988.

AMINOFF, D., OGDEN, L.L., and ROLFES-CURL, A. Isolation and characterization of human senescent erythrocytes. The Gerontologist 29, 91A, 1989.

AMINOFF, D. The molecular basis of aging and sequestration of mammalian erythrocytes. In: The Red Cell: Seventh Ann Arbor Conference. Progress in Clinical and Biological Research 319, Brewer, G.J. (Editor), pp. 247258, Alan R. Liss, Inc., New York, 1989.

ANDERSON, R.A. and LOVRIEN, R. Stoichiometry of wheat germ agglutinin as a morphology controlling agent and as a morphology protective agent for the human erythrocyte. $J$. Cell Biol. 85, 534-538, 1980.

BHAVANADAN, V.P. and KATLIC, A.W. The interaction of wheat germ agglutinin with sialoglycoproteins. The role of sialic acid. J. Biol. Chem. 254, 4000-4008, 1979.

BOYUM, A. A one-stage procedure for isolation of granulocytes and lymphocytes from human blood. Scand. $J$. Clin. Lab. Invest. Suppl. 97, 77-89, 1968.

DOCKTER, M.E. and MORRISON, M. Paroxysmal nocturnal hemoglobinuria erythrocytes are of two distinct types: Positive or negative for acetylcholinesterase. Blood 67, 540-543, 1986.

GALILI, U., RACHMILEWITZ, E.A., PELEG, A. and FLECHNER, I. A unique natural human IgG antibody with anti- $\alpha$-galactosyl specificity. J. Exp. Med. 160, 1519-1531, 1984.

GUTOWSKI, K.A., HUDSON, J.L., and AMINOFF, D. Flow cytometric analysis of human erythrocytes. I. Probed with lectins and immunoglobulins. Exp. Gerontol. 26, 315-326, 1991.

HELDRUP, J. A new technique using an aggregating antibody against glycophorin A for purging ficoll-paque separated leucocytes of contaminating erythroid lineage cells. Scand. J. Immunol. 31, 289-296, 1990.

IVATT, R.J., HARNETT, P.B., and REEDER, J.W. Isolated erythroglycans have a high-affinity interaction with wheat germ agglutinin, but a poorly accessible in situ. Biochem. Biophys. Acta 881, 124-134, 1986.

JENNINGS, L.K., BROWN, L.K., and DOCKTER, M.E. Quantitation of protein 3 content of circulating erythrocytes at the single cell level. Blood 65, 1256-1262, 1985.

JOURDIAN, G.W., DEAN, L., and ROSEMAN, S. The sialic acid. XI. A periodate-resorcinol method for the quantitative estimation of free sialic acids and their glycosides. J. Biol. Chem. 246, 430-435, 1971.

KAY, M.M.B. Role of physiologic autoantibody in the removal of senescent human red blood cells. J. Supramol. Struct. 9, 555-567, 1978.

KIM, Y.R. and ORNSTEIN, L. Isovolumetric sphering of erythrocytes for more accurate and precise cell volume measurements by flow cytometry. Cytometry 3, 419-427, 1983.

LANGOIS, R.G., BIGBEE, W.L., and JENSEN, R.H. Flow cytometric characterization of normal and variant cells with monoclonal antibodies specific for glycophorin. A. J. Immunol. 134, 4009-4014, 1985.

LOKEN, M.R., CIVIN, C.I., BIGBEE, W.L., LANGOIS, R.G., and JENSEN, R.H. Coordinate glycosylation and cell surface expression of glycophorin A during normal human erythropoiesis. Blood 70, 1959-1961, 1987.

NEWMAN, P.J., ALLEN, R.W., KAHN, R.A., and KUNICKI, T.J. Quantitation of membrane glycoprotein IIIa on intact human platelets using the monoclonal antibody, AP-3. Blood 65, 227-232, 1985.

OGDEN, L.L., ROLFES-CURL, A., and AMINOFF, D. Sequential desialylation of human erythrocytes in circulation. Proc. of Xth Intl. Symposium on Glycoconjugates (Jerusalem, Israel) 318, 19, 1989.

THOMPSON, C.B., GALLI, R.L., MELARAGNO, J., and VALERI, C.R. A method for the separation of erythrocytes on the basis of size using counterflow centrifugation. Am. J. Hematol. 17, 177-183, 1984.

VAYSSE, J., VASSY, R., ECLACHE, V., GATTEGNO, L., BLADIER, D., and PILARDEAU, P. Some characteristics of human red blood cells separated according to their size: A comparison with density fractionated red blood cells. Am. J. Hematol. 28, 232-238, 1988. 